\title{
Analysis of the Current Situation and Development of Tencent WeChat
}

\author{
Jing Lin \\ Pepperdine University, Malibu, CA 90263 \\ lesley.lin@pepperdine.edu

\begin{abstract}
This study mainly analyzed how WeChat monopolies the social media market in China. Tencent used many marketing strategies to develop WeChat, for example, the Red Pocket function was cooperating with Chinese New Year to promote; and this also helped WeChat Pay to spread because the Red Pocket is Foot-in-the-door technique. These marketing strategies are valuable to learn and discuss the positive and negative impacts on the products. By analysis WeChat's developments, it is helpful to predict the future of the social media in China and it can provide some references for other social media products.
\end{abstract}

Keywords : Tencent, WeChat, QQ, Social media, Marketing

\section{INTRODUCTION}

Nowadays, in each country, there are some popular social media platforms. For different age groups, there are different social media that design for them. For example, in the US, Instagram is for young people to post beautiful photos, Facebook is for older people to chat with each other, Snapchat is for students to send funny pictures. However, in China, there are two social media domains in the market, WeChat and QQ; also, these two applications are owned by the same company, Tencent, which is incredible. It is interesting to find out how Tencent achieves this accomplishment. By looking through Tencent's history, WeChat was the latecomer who surpassed the former. QQ was the first product that Tencent launched and it developed very well until WeChat launched. The difference between WeChat and QQ was not that obvious, but based on today's situation, WeChat is more successful than QQ. It is necessary to discuss the reasons of the success of $\mathrm{WeChat}$ and the shortcomings of QQ. By discovering the logical of the developments of WeChat and QQ, people can learn more about the social media market in China. The histories of WeChat and QQ can become useful references for other products in Chinese market. This paper will discuss about what are the functions in the WeChat and how WeChat became the top 1 social media in China. In addition, QQ as the leading social media in China before
WeChat, it will also be introduced and compared later in this paper.

\section{OVERVIEW OF TENCENT AND WECHAT}

\subsection{Tencent}

Tencent, one of the largest technology companies in China, generated around $\$ 70$ billion in income in 2020 [5]. Tencent is a digital service provider company founded in 1998. Tencent started with an application called OICQ which is the first instant-communication application. Later in 2001, Tencent launched QQ which is a social media that used in computers. QQ soon became popular among Chinese market because it is very convince for people to do office work through QQ [4]. In addiction, QQ launched many games and other functions for young people to social and relax. QQ was very successful during 2001-2012 due to QQ game, QQzone, and many other functions in QQ.

After that, Tencent launched WeChat which is a social media application that used in mobile devices, because Tencent realized that mobile phones were developed rapidly and the application market for mobile phones were empty during that time. WeChat is similar with QQ but WeChat is much simpler for user to use. The keywords for WeChat are fast, simple, and convenience. Therefore, WeChat gained many middle age users who did not like QQ because QQ is complexed 
and has too much functions. As the core products, WeChat had 1225 million monthly active users in 2020 [5]. WeChat launched in 2011, and in ten years it developed rapidly. Before WeChat launched, QQ was the top social media in China since 1999. However, WeChat beat QQ and became the top social media app in China. WeChat made many incredible decisions and changes to achieve success.

\subsection{Wechat}

In 2011, Tencent realized the future of social media in mobile electronic devices. Therefore, they launched WeChat. Even though WeChat and QQ are both social media, they targeted different markets. QQ is a social media design for computers, and it has many derivative evaluations such as QQ games, QQzone, QQshow, QQmail, etc. In other words, QQ created a digital world for people to do many things, such as dressing up in QQshow, posting comments or images in QQzone, having fun, and keeping a pet in QQgames, and so on.

On the other hand, WeChat is a social media design for mobile devices and tries to help people live easier or do things faster. WeChat connects with people's life closely, and now it has become a tool and a "social ID" that people can live much more conveniently in China. It's not only social media anymore. There was a question about how to deal with a WeChat account if its owner passed away. This question caused a massive discussion in China since WeChat can represent or record a person's whole life. In short, even though QQ and WeChat are defined as social media, they are different in function, and $\mathrm{WeChat}$ beat QQ to become the top social media app in China.

In the beginning, WeChat starred as a mini mail app. During that time, many competitors did the same thing as Tencent did. The first time when WeChat stood out was when they raised an idea about socializing with strangers. They launched a new function in WeChat called "Shake Shake," When people shake their phone, people around them will show up on their phone and chat with them. By launching this function, WeChat expanded its user number, and then they launched "Drift Bottle," which is a function that can contact strangers far away. "Simple Social and Social with Strangers" this idea helped WeChat gain a first-mover advantage.

Moreover, WeChat launched adding friends with QR Code which they call "Scan Scan." This "Scan Scan" function was new to people, which could cause people's curiosity to use this function to add friends in WeChat. WeChat gained many users through these two functions.

\section{ANALYSIS OF WECHAT FUNCTION}

\subsection{WeChat Moments function}

Once WeChat gathered enough users, they launched a function called Moments, a function similar to Facebook. As Ju Ben described: "WeChat has a function called 'Moments', where users can share photo and accompanying short text messages. This provides users with a platform to share pictures and images that present an image of the self, including what they are doing, what they like, and how they evaluate the activities, events and 'moments' of their lives; in addition, their WeChat friends may comment and send feedback.[1]" Later, People not only use this function to share their moments in their lives but also use this function to sale products. Therefore, this Moments function created a business industry in which people sell their products through WeChat. This industry is prevalent because it almost has zero cost to start a business on WeChat. Many people will use the functions that can add strangers to expand their friends in WeChat, and then they will post the pictures or videos of their product in Moments to sell. For example, people who live in foreign countries can buy the products and sell them to people who lives in China by posting products in WeChat Moments. In addition, these businesses can developed rapidly because of WeChat Pay which can transfer money through WeChat. WeChat Pay provide a more convenience environment for those business in WeChat. Since starting a business in WeChat do not need to pay for rent for store and the daily expenses in the store, the business owners in WeChat can save a lot of cost and there is almost no cost for people to start a business in WeChat. Therefore, more and more people started their business in WeChat as their main source of money. This industry is matured in China now.

\subsection{Official Account function}

WeChat also launched a function called Official Account, which is similar to Twitter. The owner of the Official Account will post articles on their account, and people can follow them to receive their updates. If the Official Account has enough followers, the owner can earn much money by operating this account through advertisements. Official Accounts provide a stage for merchants and customers to communicate.

Now Official Account is developed very well and it became a mini official website for many famous brands. Almost, all the brands in Chinese market will have a WeChat Official Account to update their new information, because the probability for people to see the information in the Official Account is much larger than the individual official website for each brand. Not only merchants will use Official Accounts to release announcements but also many organizations will use this 
function as well. For example, many news companies will release daily news in Official Accounts. Therefore, the information in Official Accounts is very diverse and comprehensive. As Lingyun Guo concluded that more than seventy precent of users appreciate WeChat because of its function of updating new information for product and all the readings that they can access.[2]" As the result, articles in Official Account satisfy people's BASIC demand for information every day. WeChat combined Twitter, Facebook, and Instagram and became an all-around social app. This is a powerful reason for people to choose to use WeChat instead of other apps.

\subsection{WeChat Pay function}

After WeChat claimed to socialize with strangers and created its information circle, WeChat made a significant change to its position. WeChat turned its placement into a more personal social app, and creating WeChat Pay lead WeChat to become a part of people's lives in China. To let people use WeChat Pay, WeChat started a function called "Red Pocket," which is based on a Chinese New Year culture. In Chinese New Year, elders will give children a Red Pocket, a red pocket with cash. This Red Pocket was extremely popular in China during the New Year season once it launched. This is "a foot in the door" technique to lead people to combine their bank account with WeChat.

Later, WeChat also asked its users to integrate their social ID to WeChat for the safety of trading through WeChat. This step that WeChat did, lead WeChat to its future success. When more and more people combined their social ID and bank account with WeChat, WeChat gained much more loyal users. By the study showed: Results from a qualitative investigation $(\mathrm{N}=70$ responses) and quantitative investigation $(\mathrm{N}=472)$ indicate that $\mathrm{WeChat}$ Pay is relatively more convenient and advantageous and also offers relatively more security and privacy protection compared to the traditional payment methods. [3]" Therefore, WeChat pay kept the advantage of WeChat which bring a much more convenience way to live and helped WeChat increased the user loyalty as well.

\subsection{Mini Program function}

After WeChat launched many basic function to increase the conveniency of people's daily life, WeChat also launched Mini Program function which is a way to approach other functions quickly and easily such as playing games, creating survey, ordering food, online shopping, etc. By using Mini Program, users do not to login anymore because it will help users to login with their WeChat accounts automatically. This function pushes the advantage of $\mathrm{WeChat}$ into another higher level. For example: "an SoC (system-on-chip) and WeChat mini-program that focuses on low-cost hardware, rapid development, user-friendly application design, and helping developers get a quick start in building a DIY monitoring system. The ESP8266, a high-performance SoC, is used as the microcontroller and Wi-Fi module to transfer the sensor data to a remote server. A WeChat mini-program provides the graphical user interface, enabling users to manage devices and access data by clicking. Users can log into the system using their WeChat accounts and bind devices by scanning QR codes on the devices. Thus, the complex management and device binding in conventional systems can be overcome. The system is easy to be expand and has great potential for greenhouse environmental monitoring in China. [6]" Therefore, WeChat became the must-downland application for mobile phones in China. WeChat has almost all the functions that people need for their daily life and moreover these functions are not complexity at all. This is the best accomplishment that WeChat did, they used the simplest way to help people do the most things.

\section{THE SUCCESS FACTORS OF WECHAT}

WeChat is a service for Chinese who are in China and for Chinese who are outside of China. Using WeChat, people can FaceTime, Call, or text with friends without geographical limits for free. In other words, WeChat combines the function of Facebook into it as well. People outside of China will still have friends or families in China, so WeChat becomes the best choice for them to contact their friends and families.

WeChat can be successful because WeChat never stops. WeChat Pay became a necessary tool in people's life because the WeChat team kept accepting new things and adapting to the social trend. WeChat is cooperating with other companies to increase the frequency of the use of WeChat Pay. For example, WeChat collaborated with DiDi, which is an app similar to Uber. DiDi is the most popular taxi-hailing app. Also, the most popular take-out apps are cooperating with WeChat Pay. Since the WeChat team never stopped updating their app and expanding their influence, WeChat Pay collaborated with almost all the apps needed to deal with online payment. As WeChat Pay grew, WeChat also charged service fees from money transferring.

WeChat has many functions which sound completed. However, the critical words for WeChat are simple, easy, and convenient. This is the most fantastic technique that WeChat has. WeChat can combine all the functions that people need in real life most simply. Moreover, WeChat loves new things, and they will accept all the new items and convert them into a more straightforward way and blend them into WeChat.

WeChat is always synchronous with the social environment and improves itself all the time. These two characteristics may be the key to the success of WeChat. 
WeChat can beat QQ only because WeChat connects with people's real life. Therefore, WeChat can suit a much more extensive range of people than QQ does. There will still be a group of people who love QQ Games or QQzone stick with QQ. Therefore, even though WeChat is more popular than QQ now, QQ will not disappear. One is a QQ empire containing many people's digital lives, and another one is an excellent life help that blends in people's real lives. This study is based on the information on Tencent's official website, so the information is limited. The marketing techniques that WeChat used were very valuable for people to study. WeChat is one of the most successful products in Chinese market. In the future, I will look more into Tencent's marketing strategies and beads on WeChat and QQ's history. We can learn more about Chinese's preference. Moreover, we can predict a product's tendency and improve the product to become more suitable for Chinese market.

\section{CONCLUSION}

Overall, this paper summaries the history and functions of WeChat, and analysis the success of WeChat. In the aspect of functions of WeChat, its moment, payment, subscription service, and mini program are our daily common things. As for its success fators, it is necessary to mention its continuous creative spirits and being a listener to all sounds whether it is good or bad. Furthermore, WeChat is always glad to make all the functions more accessible to our daily communication. Nevertheless, there still are many data and information that need to be improve in order to achieve a better result. Based on the information above, WeChat's status in China cannot be replaced in recent years.

\section{ACKNOWLEDGMENTS}

It is my great pleasure to pay a heart-felt gratitute to my teachers and supervisor who guided my paper during this period. My paper cannot be finished without their help.

Besides, I would like to thank my parents and my friends who supported me and comforted me when i was in a down spirit.

Finally, I am glad to have readers as supervisors to give me suggestion whenever it is possible.

\section{REFERENCES}

[1] Bei, J. (2017). A case study of Foreigner's INTERCULTURAL identity in China via WECHAT MOMENTS. SHS Web of Conferences, 33, 00031.
[2] Guo, L., Zhang, M., Kang, K., \& Hu, M. (2017) Transforming followers into fans: A study of Chinese users of the WECHAT official account. Online Information Review, 41(7), 1029-1045. https://doi.org/10.1108/oir-01-2016-0013

[3] Mombeuil, C., \& Uhde, H. (2021). Relative convenience, relative Advantage, PERCEIVED security, perceived privacy, and continuous USE intention of China's WeChat pay: A Mixed-method TWO-PHASE design study. Journal of Retailing and Consumer Services, 59, 102384. https://doi.org/10.1016/j.jretconser.2020.102384

[4] Negro, G., Balbi, G., \& Bory, P. (2020). The path to WECHAT: HOW TENCENT'S culture shaped the most popular CHINESE APP, 1998-2011. Global Media and Communication, 16(2), 208-226. https://doi.org/10.1177/1742766520923008

[5] "Tencent Financial Releases.” Investors - Tencent , www.tencent.com/en-us/investors.html.

[6] Zhou, L., Qiu, Z., \& He, Y. (2020). Application of Wechat Mini-program and WI-FI SoC in AGRICULTURAL IoT: A LOW-COST greenhouse monitoring system. Transactions of the ASABE, 63(2), 325-337. https://doi.org/10.13031/trans.13499

https://doi.org/10.1051/shsconf/20173300031 